\title{
Differential expression of the cationic amino acid transporter 2(B) in the adult rat brain.
}

\author{
Olivier Braissant ${ }^{1}$, Tomomi Gotoh ${ }^{2}$, Marc Loup ${ }^{1}$, \\ Masataka Mori ${ }^{2}$ and Claude Bachmann" ${ }^{1}$
}

1 Central Clinical Chemistry Laboratory, University Hospital, CH-1011 Lausanne, Switzerland.

${ }^{2}$ Department of Molecular Genetics, Kumamoto University School of Medicine, Honjo 2-2-1, Kumamoto 860-0811, Japan.

16 pages, including 2 tables and 2 figures.

\section{Correspondence to :}

Olivier Braissant

Central Clinical Chemistry Laboratory,

University Hospital,

CH-1011 Lausanne, Switzerland

Tél : (+41.21) 314.41.52

Fax : (+41.21) 314.42.88

e-mail : Olivier.Braissant@chuv.hospvd.ch 


\begin{abstract}
L-arginine is a substrate for the synthesis of proteins, nitric oxide (NO), creatine, urea, proline, glutamate, polyamines and agmatine. In the central nervous system (CNS), arginine is extracted from the blood and exchanged by cells through carriers called cationic amino acid transporters (CAT) and belonging to the so-called system $\mathrm{y}^{+}$. In order to better understand the arginine transport in CNS, we studied in detail the regional distribution of the cells expressing the CAT2(B) transcript in the adult rat brain by non-radioisotopic in situ hybridization. We show that CAT2(B) is expressed in neurons and oligodendrocytes throughout the brain, but is not detected in astrocytes. The pattern of localization of CAT2(B) in the normal adult rat brain fits closely that of CRT1, a specific creatine transporter. Our study demonstrates that the in vivo expression of CAT2(B) differs from the one reported in vitro, implying that local cellular interactions should be taken into account in studies of gene regulation of the CAT2(B) gene. Our work suggests that CAT2(B) may play a role in case of increased NO production as well as arginine or creatine deficiency in the brain.
\end{abstract}

Keywords: brain, arginine, cationic amino acid transporter, system $y^{+}$, nitric oxide, creatine, in situ hybridization.

Theme B: Cellular and Molecular Biology.

Topic: Gene structure and function: general. 


\section{1: Introduction}

L-arginine is a substrate for many different pathways, including nitric oxide (NO), creatine, urea, ornithine, polyamines, glutamate, proline and agmatine, and is essential for protein synthesis [29]. In the central nervous system (CNS), arginine originates from the blood, from protein breakdown or is recycled by argininosuccinate synthetase (AS) and lyase (AL) from the citrulline generated by nitric oxide synthase (NOS).

Within the brain, specific transporters designated as system y catalize the exchange of arginine between the different cell types [18]. The system $\mathrm{y}^{+}$was identified at the blood brain barrier (BBB) [27], in primary cultured astrocytes [24], in cultured neurons from neocortex and cerebellum [28], as well as in synaptosomes extracted from cortex and cerebellum [1]. System $y^{+}$carriers are a family of cationic amino acid transporters called CATs [18]. Up to now, five different CAT proteins have been identified (see [19] for review): CAT1 is considered to be ubiquitously expressed except in liver [15]; CAT2(B) is present in different tissues including brain but absent from liver [17]; CAT2A, an alternatively spliced variant of CAT2(B), is liver specific [7]; CAT3 is restricted to brain and embryonic mesoderm [9,12]; finally CAT4 is expressed in placenta, testis and at a low level in brain $[2,25]$. CAT1, CAT2(B), CAT3 and CAT4 have been described in whole brain homogenates $[9,12,15,17,25]$. We and others have recently described the precise cellular distribution of CAT1 and CAT3 in the adult rat brain [5,10]. CAT2(B) was reported to be expressed at very low levels in rat primary astrocytes [26]. CAT1, CAT2(B) and CAT3 have high affinities for arginine (apparent $\mathrm{Km}$ around $100 \mu \mathrm{M}$ in vitro), whereas the liver specific CAT2A has a much lower affinity (apparent $\mathrm{Km}$ of 2 to $5 \mathrm{mM}$ ). No data have been reported so far on the affinity of CAT4 for arginine.

For assessing the physiological impact of the different CAT proteins on NO production or creatine synthesis in CNS, the regulation of their gene expression is probably as relevant, if not more, as the affinity of the carriers for arginine in vitro. In order to understand the intercellular trafficking of arginine in the CNS from capillaries and their adjacent astrocytes to other glial cells and neurons, data on the detailed localization of CAT2(B) in the CNS are needed. Since these are missing, we investigated the expression of CAT2(B) mRNA in the adult rat brain. We have used a new combination of non-radioisotopic in situ hybridization (ISH) with immunohistochemistry to identify the cells expressing CAT2(B), and shown that it is restricted to neurons and oligodendrocytes.

\section{2: Materials and Methods}


A specific cDNA fragment for the rat CAT2 ([16]; nt 1289-1834) was obtained by reverse transcription coupled to PCR using rat liver total RNA as template, and cloned in the vector pGEM3Zf(+) into the HincII site. The resulting plasmid was linearized with HindIII to yield the antisense probe, and with XbaI to yield the sense probe. The linearized plasmids were gel-purified and used as templates for digoxigenin labelled riboprobe synthesis, using T7- or SP6-RNA polymerases (Promega, Madison, WI), as described previously [3,5]. Four female adult Wistar rats (300 g, BRL, Basel, Switzerland) were deeply anesthetized (10 mg/rat Ketalar, Parke Davis, USA) and sacrificed by decapitation. Their brain was extracted within 2 minutes, immediately embedded in tissue freezing medium (Jung, Nussloch, Germany) and frozen in isopentane and dry ice. Brains were kept at -80 ${ }^{\circ} \mathrm{C}$ until used, then cut and analyzed by non-radioactive ISH using the active-DEPC method as described $[3,5,6]$. The specificity of hybridization was ascertained by the use of a sense probe having the same length, GC content and activity of digoxigenin labelling as the corresponding antisense probe. In each ISH experiment, a section hybridized with a specific antisense probe was always preceded or followed by an adjacent section hybridized with the corresponding sense control probe. In each ISH experiment, 3 out of 4 of the antisense ISH stained sections were further processed for immunohistochemistry. On these sections, neurons, astrocytes and oligodendrocytes were labelled using monoclonal antibodies directed against microtubule associated protein 2 (MAP2), glial fibrillary acidic protein (GFAP) and myelin basic protein (MBP), as described previously [6]. The double stained sections (blue signal for ISH and red signal for immunohistochemistry) were mounted in glycerol. Sections were observed and photographed on an Olympus BX50 microscope equipped with a DP-10 digital camera (Olympus Opticals, Japan). No discrepancies were observed between the four female rats analyzed. Brain structures were identified according to Paxinos and Watson [20]. 


\section{3: Results}

The CAT2(B) mRNA was found in neurons and oligodendrocytes throughout the brain, but never in astrocytes. Its main cellular localization was perinuclear. A semi-quantitative description of CAT2(B) mRNA levels in the different brain structures is given in Table 1 . The specificity of hybridization was ascertained by the absence of signal obtained with the CAT2(B) sense probe (Fig.1g).

In the telencephalon, CAT2(B) was expressed in the neocortex, in pyramidal and non-pyramidal neurons (Fig.1a,b; co-labelling with MAP2). CAT2(B) was never detected in astrocytes, including those contacting capillary and blood vessel endothelial cells (BBB), as illustrated by a co-labelling with GFAP (Fig. 2d,f; arrowheads). In the hippocampus, the CAT2(B) transcript was localized at high levels in the CA1 layer and the dentate gyrus (Fig.2c). The CA3, polymorphic and molecular layers presented a lower signal. In the corpus callosum, CAT2(B) was located in oligodendrocytes as demonstrated by a co-labelling with MBP (Fig.2e). The CAT2(B) mRNA was well expressed in the olfactory bulb (Fig.1f) and also located in the putamen, the globus pallidum, the nucleus accumbens, the pyriform cortex and its transition towards the amygdala nuclei. Most structures of the diencephalon, midbrain, pons and medulla showed the CAT2(B) transcript at a weak level, except for the brainstem with higher signals in the vestibular nuclei, the locus coeruleus, the trigeminal nuclei and the solitatry tract nucleus. In the cerebellar cortex, CAT2(B) was abundantly expressed in neurons of the granular layer (Fig.2a-c). Purkinje cells were positive for CAT2(B) mRNA, but at a very low level of detection, as shown by a co-labelling with MAP2 (Fig.2b). Bergmann glial cells were negative, as shown by a co-labelling with GFAP (Fig.2c). CAT2(B) was also found in the cerebellar neurons of the molecular layer and deep nuclei. The CAT2(B) mRNA was expressed at a very low level of detection in the white matter structures of cerebellum, in cells identified as oligodendrocytes by a co-labelling with MBP (data not shown). The CAT2(B) transcript was also revealed in the choroid plexus (Fig.2a) and the ependymal epithelium (data not shown). 


\section{4: Discussion}

\section{1: Expression of the CAT2(B) mRNA in the brain}

Previous reports have described the presence of the CAT2(B) mRNA in total homogenates of the rodent brain [17], but without a distinction of its expression among cell types. We show in this study that in normal conditions the CAT2(B) mRNA is found both in neurons and oligodendrocytes of the whole brain. However, we could never detect its expression in astrocytes, including those contacting endothelial cells of capillaries and participating to BBB. As for CAT1, the CAT2(B) mRNA was mainly localized in the perinuclear region of the brain cells; this is in contrast with the CAT3 transcript, which we have also found in the neuronal processes [5].

A comparison of the cerebral expression of the CAT2(B) mRNA with the previously described expression of CAT1 and CAT3 transcripts [5] is given in Table 2. Our studies suggest that the prime candidate for basic arginine trafficking between brain cells is CAT1, as it is ubiquitously expressed in CNS, including at the BBB $[5,40]$. CAT2(B) and CAT3 could be more specifically involved in the arginine uptake and exchange by neurons and oligodendrocytes (CAT2(B)) and neurons only (CAT3) (this study, and [5,10]).

\section{2: Arginine uptake and metabolism in brain cells}

The availability and uptake of arginine for the different types of brain cells is a prerequisite for their local NO and creatine synthesis. Evidence is growing that depending on the physiological and/or pathological conditions to which the CNS is exposed, the $\mathrm{y}^{+}$system of arginine transport as well as the intracellular metabolism and recycling of arginine can be regulated at the molecular level.

Rats injected intraperitoneally with lipopolysaccharide (LPS) present a dose-dependent induction of the inductible and endothelial NOS genes in the brain, which is restricted to astrocytes [13]. It was also shown that primary rat astrocytes treated by LPS and interferon- $\gamma$ (IFN $\gamma)$ present an induction of the CAT2(B) gene, together with an increase of their arginine uptake, an induction of the iNOS gene and an increase of their NO synthesis [26]. In the same study, primary neurons did not express CAT2(B) in control conditions, and did not induce its expression under LPS and IFN $\gamma$ treatment. Neuronal PC12 cells do not express CAT2(B) either constitutively or under IFN $\gamma$ and tumor 
necrosis factor $\alpha(\mathrm{TNF} \alpha)$ treatment, whereas they show an induction of the iNOS gene [31]. As CAT2(B) is not expressed in vitro in primary neurons nor in PC12 cells [26,31], the constitutive neuronal expression of CAT2 we found in this study indicates that the normal brain differs from the conditions to which single cell type cultures are exposed in vitro. The CAT2(B) expression in the CNS, with its different cell types, might thus depend on intercellular interactions.

The intracellular recycling of arginine by AS and AL has also been shown to be regulated: LPS and IFN $\gamma$ injected into the rat brain induce the AS gene in glial cells [8], whereas TNF $\alpha$ and IFN $\gamma$ induce both the AS and AL genes in PC12 cells [31]. In human and mouse, primary or secondary defects of urea cycle activity lead to a significant decrease of blood arginine concentration $[11,14,22,23]$. This is also found in portacaval shunted or ammonium chloride-treated rats which show, in synaptosomes prepared from different parts of the brain, an increase of arginine uptake in parallel with augmented NOS activity [21]. We have recently observed that the CNS expression of the arginine recycling genes $\mathrm{AS}$ and $\mathrm{AL}$ is enhanced in astrocytes under ammonium chloride treatment [4]. Thus the arginine transport and metabolism seems affected by increased ammonia.

The arginine transport might also be relevant for the creatine synthesis in CNS. Recent studies suggest that in normal conditions the brain might depend on its own creatine synthesis due to the absence of a creatine transport system at the BBB [6,30]. In consequence, brain cells synthesizing creatine depend on the uptake of arginine from the blood and on its local trafficking between the different cell types, and on the CNS expression of the CAT genes. Interestingly, the differential pattern of expression of CAT2(B) in the rat brain closely follows the expression of CRT1, a specific transporter for creatine (see Table 2, and details in [6]). One might speculate that in the brain the expression of CAT2(B) and CRT1 is coordinated, and furthermore that CAT2(B) might be involved in the creatine synthesis pathway in the CNS.

In the non-nervous tissues, numerous factors have been reported that regulate the CAT genes at the molecular level, including cytokines, growth factors, hormones, LPS or a low protein diet (see $[18,19]$ for reviews and references therein). In the CNS or in cell cultures issued from the nervous tissue however, only a few data are available. The CAT1 mRNA is constitutively expressed in neuronal PC12 cells as well as in primary neurons and astrocytes. However it is not regulated by 
LPS, IFN $\gamma$ nor TNF $\alpha$ treatments, nor is CAT3 induced in PC12 cells by TNF $\alpha$ and IFN $\gamma[26,31]$. To date in CNS, the only CAT gene which has a regulated expression appears to be CAT2(B): it is inducible in primary astrocytes under a LPS and IFN $\gamma$ treatment [26]. It remains to be shown if the induction of CAT2(B) also plays a role in vivo, e.g. under pathological conditions. In particular, it could be relevant to test if the brain is able to increase its extraction of arginine from the blood by induction of the CAT2(B) gene in conditions of arginine or creatine deficiency. 


\section{References}

[1] Aldridge, C.R. and Collard, K.J., The characteristics of arginine transport by rat cerebellar and cortical synaptosomes, Neurochem.Res., 21 (1996) 1539-1546.

[2] Ayuk, P.T., Sibley, C.P., Donnai, P., D'Souza, S. and Glazier, J.D., Development and polarization of cationic amino acid transporters and regulators in the human placenta, Am.J.Physiol. Cell Physiol., 278 (2000) C1162-C1171.

[3] Braissant, O. and Wahli, W., A simplified in situ hybridization protocol using non-radioactively labelled probes to detect abundant and rare mRNAs on tissue sections, Biochemica, 1 (1998) $10-16$.

[4] Braissant, O., Honegger, P., Loup, M., Iwase, K., Takiguchi, M. and Bachmann, C., Hyperammonemia: regulation of argininosuccinate synthetase and argininosuccinate lyase genes in aggregating cell cultures of fetal rat brain, Neurosci.Lett., 266 (1999) 89-92.

[5] Braissant, O., Gotoh, T., Loup, M., Mori, M. and Bachmann, C., L-arginine uptake, the citrulline-NO cycle and arginase II in the rat brain: an in situ hybridization study, Mol.Brain Res., 70 (1999) 231-241.

[6] Braissant, O., Henry, H., Loup, M., Eilers, B. and Bachmann, C., Endogenous synthesis and transport of creatine in the rat brain: an in situ hybridization study, Mol.Brain Res., in press.

[7] Closs, E.I., Albritton, L.M., Kim, J.W. and Cunningham, J.M., Identification of a low affinity, high capacity transporter of cationic amino acids in mouse liver, J.Biol.Chem., 268 (1993) 7538-7544.

[8] Heneka, M.T., Schmidlin, A. and Wiesinger, H., Induction of argininosuccinate synthetase in rat brain glial cells after striatal microinjection of immunostimulants, J.Cer.Blood Flow Metab., 19 (1999) 898-907.

[9] Hosokawa, H., Sawamura, T., Kobayashi, S., Ninomiya, H., Miwa, S. and Masaki, T., Cloning and characterization of a brain-specific cationic amino acid transporter, J.Biol.Chem., 272 (1997) 8717-8722.

[10] Hosokawa, H., Ninomiya, H., Sawamura, T., Sugimoto, Y., Ichikawa, A., Fujiwara, K. and Masaki, T., Neuron-specific expression of cationic amino acid transporter 3 in the adult rat brain, Brain Res., 838 (1999) 158-165. 
[11] Inoue, I., Gushiken, T., Kobayashi, K. and Saheki, T., Accumulation of large neutral amino acids in the brain of sparse-fur mice at hyperammonemic state, Biochem.Med.Met.Biol., 38 (1987) 378-386.

[12] Ito, K. and Groudine, M., A new member of the cationic amino acid transporter family is preferentially expressed in adult mouse brain, J.Biol.Chem., 272 (1997) 26780-26786.

[13] Iwase, K., Miyanaka, K., Shimizu, A., Nagasaki, A., Gotoh, T., Mori, M. and Takiguchi, M., Induction of endothelial nitric-oxide synthase in rat brain astrocytes by systemic lipopolysaccharide treatment, J.Biol.Chem., 275 (2000) 11929-11933.

[14] James, J.H., Escourrou, J. and Fischer, J.E., Blood-brain neutral amino acid transport activity is increased after portacaval anastomosis, Science, 200 (1978) 1395-1397.

[15] Kim, J.W., Closs, E.I., Albritton, L.M. and Cunningham, J.M., Transport of cationic amino acids by the mouse ecotropic retrovirus receptor, Nature, 352 (1991) 725-728.

[16] MacLeod, C.L., Finley, K., Kakuda, D., Kozak, C.A. and Wilkinson, M.F., Activated T cells express a novel gene on chromosome 8 that is closely related to the murine ecotropic retroviral receptor, Mol Cell Biol., 10 (1990) 3663-3674.

[17] MacLeod, C.L., Finley, K.D. and Kakuda, D.K., y(+)-type cationic amino acid transport: expression and regulation of the mCAT genes, J.Exp.Biol., 196 (1994) 109-121.

[18] Malandro, M.S. and Kilberg, M.S., Molecular biology of mammalian amino acid transporters, Ann.Rev.Biochem., 65 (1996) 305-336.

[19] Palacin, M., Estevez, R., Bertran, J. and Zorzano, A., Molecular biology of mammalian plasma membrane amino acid transporters, Physiol.Rev., 78 (1998) 969-1054.

[20] Paxinos, G. and Watson, C. (Eds), The rat brain in stereotaxic coordinates, Vol. 2nd, Academic Press Limited, London, 1986, 260 pp.

[21] Rao, V.L., Audet, R.M. and Butterworth, R.F., Increased nitric oxide synthase activities and L-[3H]arginine uptake in brain following portacaval anastomosis, J.Neurochem., 65 (1995) 677-678.

[22] Ratnakumari, L., Qureshi, I.A. and Butterworth, R.F., Regional amino acid neurotransmitter changes in brains of spf/Y mice with congenital ornithine transcarbamylase deficiency, Metab.Brain Dis., 9 (1994) 43-51.

[23] Ratnakumari, L., Qureshi, I.A., Butterworth, R.F., Marescau, B. and De, D.P., Argininerelated guanidino compounds and nitric oxide synthase in the brain of ornithine 
transcarbamylase deficient spf mutant mouse: effect of metabolic arginine deficiency, Neurosci.Lett., 215 (1996) 153-156.

[24] Schmidlin, A. and Wiesinger, H., Stimulation of arginine transport and nitric oxide production by lipopolysaccharide is mediated by different signaling pathways in astrocytes, J.Neurochem., 65 (1995) 590-594.

[25] Sperandeo, M.P., Borsani, G., Incerti, B., Zollo, M., Rossi, E., Zuffardi, O., Castaldo, P., Taglialatela, M., Andria, G. and Sebastio, G., The gene encoding a cationic amino acid transporter (SLC7A4) maps to the region deleted in the velocardiofacial syndrome, Genomics, 49 (1998) 230-236.

[26] Stevens, B.R., Kakuda, D.K., Yu, K., Waters, M., Vo, C.B. and Raizada, M.K., Induced nitric oxide synthesis is dependent on induced alternatively spliced CAT-2 encoding L-arginine transport in brain astrocytes, J.Biol.Chem., 271 (1996) 24017-24022.

[27] Stoll, J., Wadhwani, K.C. and Smith, Q.R., Identification of the cationic amino acid transporter (System y+) of the rat blood-brain barrier, J.Neurochem., 60 (1993) 1956-1959.

[28] Westergaard, N., Beart, P.M. and Schousboe, A., Transport of L-[3H]arginine in cultured neurons: characteristics and inhibition by nitric oxide synthase inhibitors, J.Neurochem., 61 (1993) 364-367.

[29] Wu, G.Y. and Morris, S.M., Arginine metabolism: nitric oxide and beyond, Biochem.J., 336 (1998) 1-17.

[30] Wyss, M. and Kaddurah-Daouk, R., Creatine and creatinine metabolism, Physiol.Rev., 80 (2000) 1107-1213.

[31] Zhang, W.Y., Gotoh, T., Oyadomari, S. and Mori, M., Coinduction of inducible nitric oxide synthase and arginine recycling enzymes in cytokine-stimulated PC12 cells and high output production of nitric oxide, Mol.Brain Res., 83 (2000) 1-8. 


\section{Figure legends:}

Figure 1: Expression of the CAT2(B) mRNA in the telencephalon. a-f: ISH antisense staining for CAT2(B) (blue signal), co-labelled by immunohistochemistry (red signal) for MAP2 (neurons, a,b,c), GFAP (astrocytes, d,f) and MBP (oligodendrocytes, e). g: : ISH sense staining for CAT2(B). a,b: Layer V of neocortex (pyramidal neurons: arrowheads, non-pyramidal neurons: asterisk). c: Dentate gyrus of hippocampus. d: Blood-brain barrier in neocortex with astrocytes (arrowheads) extending their podocytes towards capillaries. e: White matter of corpus callosum (oligodendrocytes: arrowhead). f,g: Olfactory bulb. Bar: $50 \mu \mathrm{m}$.

Figure 2: Expression of the CAT2(B) mRNA in the cerebellar cortex. ac: ISH antisense staining for CAT2(B) (blue signal), co-labelled by immunohistochemistry (red signal) for MAP2 $(\mathbf{a}, \mathbf{b})$ and GFAP (c). a: Cerebellar cortex with the molecular layer (M), the Purkinje cell layer $(\mathbf{P})$ and the granule cell layer (G). Choroidal plexus: asterisk. b,c: Higher magnification of the area indicated in panel a, with Purkinje cells (b) and Bergman glia (c) located by arrowheads. Bar: 50 $\mu \mathrm{m}$. 
Table 1: Differential expression of the CAT2(B) mRNA in the adult rat brain.

\begin{tabular}{|c|c|c|c|}
\hline Telencephalon & & Hypothalamus (continuation) & \\
\hline Neocortex & & Premammillary ventral nucleus & + \\
\hline Pyramids of layers III and V & ++ & Zona incerta & + \\
\hline Other neurons & + & Preoptic area & + \\
\hline Astrocytes & - & Pituitary (posterior lobe) & + \\
\hline \multicolumn{4}{|l|}{ Corpus callosum } \\
\hline Oligodendrocytes & + & Midbrain & \\
\hline Astrocytes & - & Superior colliculus & + \\
\hline Olfactory bulb & +++ & Inferior colliculus & + \\
\hline Anterior olfactory nucleus & + & Red nucleus & \pm \\
\hline Anterior commissure & \pm & Substantia nigra & \pm \\
\hline Caudate putamen & + & Retrorubral fields & \pm \\
\hline Amygdala & + & & \\
\hline Island of Calleja & ++ & Brainstem & \\
\hline Nucleus accumbens & ++ & Pontine nucleus & + \\
\hline Pyriform cortex & + & Trapezoid body nucleus & + \\
\hline Hippocampus & & Vestibular nuclei & ++ \\
\hline CA1 & ++ & Tegmental nuclei & + \\
\hline CA3 & + & Locus coeruleus & ++ \\
\hline Dentate gyrus & +++ & Subcoeruleus nuclei & + \\
\hline Polymorphic / & + & Reticulate formation & + \\
\hline \multicolumn{4}{|l|}{ molecular layers } \\
\hline Septal nuclei & + & Inferior olive & \pm \\
\hline Diagonal band & + & Trigeminal nuclei & ++ \\
\hline Globus pallidum & + & Solitary tract nucleus & +++ \\
\hline Diencephalon & & Cerebellum & \\
\hline Thalamus & & Molecular layer & + \\
\hline Stria medullaris & \pm & Purkinje cells & + \\
\hline Laterodorsal nucleus & + & Bergmann glia & - \\
\hline Lateroposterior nucleus & + & Granular layer neurons & ++++ \\
\hline Lateroventral nucleus & + & Cerebellar white matter & \\
\hline Ventromedial nucleus & + & Oligodendrocytes & \pm \\
\hline Posterior group nuclei & + & Astrocytes & - \\
\hline Anterior pretectal nucleus & + & Deep nuclei neurons & + \\
\hline Reticular nucleus & + & & \\
\hline Hypothalamus & & Choroidal plexus & + \\
\hline Lateral hypothalamic area & + & Ependymal / subependymal layers & + \\
\hline
\end{tabular}

-: absent; \pm : barely detectable; +: weak expression; ++: moderate expression; +++: strong expression; ++++: very strong expression. The semi-quantitative description of CAT2(B) mRNA levels is based on ISH only experiments. Levels of transcripts observed by optical microscopy are indicated by - and + signs, which do not represent a strict linear measure of mRNA levels. 
Table 2: Summary expression of CAT1, CAT2(B), CAT3 and CRT1 in the rat brain.

\begin{tabular}{l|ccc|c} 
& CAT1 & CAT2(B) & CAT3 & CRT1 \\
\hline Neurons & + & + & + & + \\
Oligodendrocytes & + & + & - & + \\
Astrocytes & + & - & - & -
\end{tabular}

CAT: Cationic amino acid transporter; CRT1: creatine transporter. The expression data for CAT1, CAT3 and CRT1 are taken from [5,6]. 



Braissant et al., CAT2(B)

Figure 1 

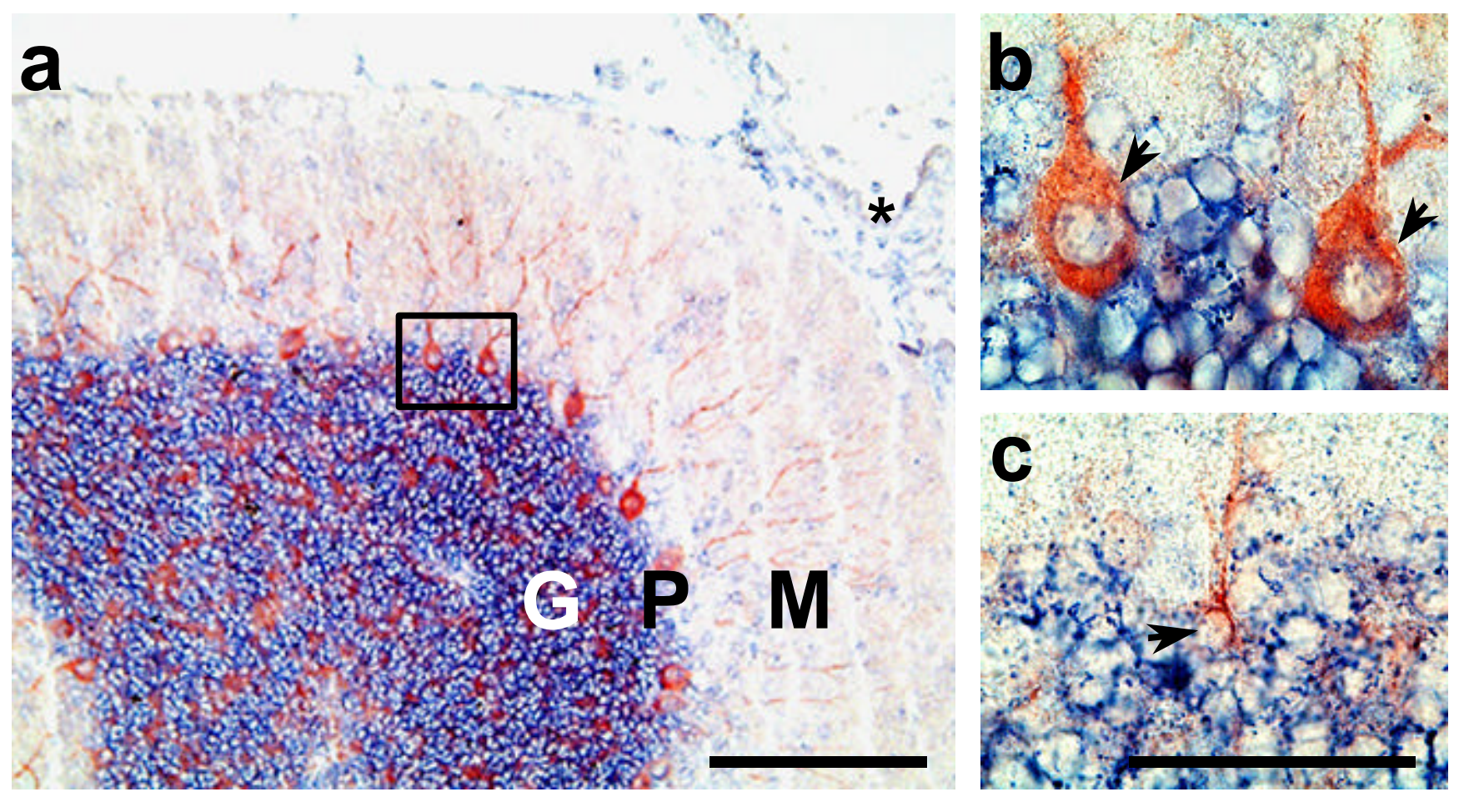

Braissant et al., CAT2(B)

Figure 2 